A C G

Rec. Nat. Prod. 13:1 (2019) 37-49

records of natural publications products

\title{
HPLC Fingerprint of Important Phenolic Compounds in Some Salvia L. Species from Iran
}

\author{
Marzieh Fotovvat $^{\oplus 1,4}$, Tayebeh Radjabian ${ }^{{ }^{*} 1,2}$ and Azra Saboora ${ }^{\oplus 3}$
}

\author{
${ }^{1}$ Department of Biology, Faculty of Basic Sciences, Shahed University, Tehran, Iran \\ ${ }^{2}$ Immunoregulation Research Center, Shahed University, Tehran, Iran \\ ${ }^{3}$ Department of Plant Sciences, Faculty of Biological Sciences, Alzahra University, Tehran, Iran \\ ${ }^{4}$ Department of Plant Sciences, Faculty of Biological Sciences, Kharazmi University, Tehran, Iran
}

(Received February 13, 2018; Revised April 27, 2018; Accepted April 27, 2018)

\begin{abstract}
Salvia L. is a large genus of the Lamiaceae family with high medicinal value. Pharmaceutical properties of Salvia species are mainly due to their secondary metabolites, especially phenolic compounds. This study was focused on identification and determination of five bioactive phenolic compounds (rosmarinic acid, carnosic acid, caffeic acid, salvianolic acids A and B) in the 41 populations from 27 wild Salvia species of Iran using a simple and reliable HPLC-UV method. The principal component analysis (PCA) technique was used to study differentiation among species according to their phenolic compound profiles. Significant intra- and interspecific variations were observed in the distribution patterns and contents of phenolic compounds in the studied Salvia species. As a result of this study, it was found that leaves had greater amounts of phenolic compounds as compared to the roots. The highest content of rosmarinic acid $(41.53 \pm 0.88 \mathrm{mg} / \mathrm{g} \mathrm{DW})$ and salvianolic acid A $(8.10 \pm 0.35 \mathrm{mg} / \mathrm{g} \mathrm{DW})$ were found in the leaves of $S$. verticillata. The leaves of $S$. syriaca and $S$. sharifii were rich in salvianolic acid B $(54.47 \pm 2.00 \mathrm{mg} / \mathrm{g}$ DW) and carnosic acid $(34.05 \pm 1.18 \mathrm{mg} / \mathrm{g} \mathrm{DW})$, respectively. The PCA results revealed chemical variations in the Salvia species collected from different regions and could fully distinguish between them based on the phenolic compounds concentrations. The present study demonstrated that apart from $S$. officinalis, some wild species such as $S$. verticillata, $S$. hypoleuca, S. leriifolia and $S$. virgata can be introduced as potent natural sources for medicinal and industrial purposes.
\end{abstract}

Keywords: Salvia species; rosmarinic acid; salvianolic acid A; salvianolic acid B; carnosic acid; HPLC. C 2018 ACG Publications. All rights reserved.

\section{Introduction}

The genus Salvia L. (sage) consists of about 900 plant species and represents one of the most important and the largest genera of the Lamiaceae family [1-3]. Iran with 58 species, of which 17 are endemic [1-3], is one of the best countries for growing Salvia species in the world [1]. The name of Salvia comes from the Latin words salvare, salveo, salvus or salvere meaning healing, non-harmful and safe and refers to the numerous medicinal applications of Salvia species [1,3-6].

Salvia species are known for their several therapeutic properties in folk medicine to treat tuberculosis, bronchitis, pyretic, rheumatoid arthritis, colds, wounds and skin infections, headache, cerebral ischemia and memory disorders, as well as hepatitis [1,7]. In Iranian traditional medicine, different parts of many indigenous Salvia species such as S. aethiopis, S. aegyptiaca, S. officinalis, $S$. hydrangea, S. sclarea, $S$. macrosiphon and $S$. viridis are used locally for treatment of some diseases

* Corresponding author: E-Mail: rajabian@shahed.ac.ir ; Phone: 098-021-51212244 Fax: 098-021-51212201

The article was published by ACG Publications

http://www.acgpubs.org/journal/records-of-natural-products (C) January-February 2019 EISSN:1307-6167

DOI: http://doi.org/10.25135/rnp.72.18.02.228 
including flatulence, eye disorders, antiseptic for wounds, diuretic and fever [8]. Furthermore, some pharmacological activities such as antioxidant, antibacterial, antifungal, antimicrobial, antitumor, antidiabetic, antituberculosis, antiplasmodial, antiinflammatory and anticholinesterase properties (treatment of Alzheimer's Disease) have been recently proven for the Salvia species through clinical studies in modern medicine $[1,9,10]$. Salvia species, specially $S$. officinalis have been traditionally used as herbal tea for the treatment of digestive and circulation disturbances, bronchitis, cough, asthma, angina, mouth and throat inflammations, depression, excessive sweating, skin ailments and as well as flavoring agents in the food industries, cosmetic, pharmaceutical and fragrance products $[9,11]$.

Members of Salvia genus have been the subjects of extensive studies with the aim of identification and characterization of potential bioactive compounds. Terpenoids (di-and triterpenoids), phenolic acid derivatives and flavonoids are the predominant secondary metabolite constituents of Salvia species [12,13]. Salvia species mainly contain two major types of biologically active compounds: lipid-soluble abietane-type diterpenoid tanshinones [14] and carnosic acid [15] and water-soluble phenolic acids and flavonoids. Phenolic acids which are widely distributed in plant species are responsible for their various therapeutic effects [14].<smiles>O=C(O)/C=C/c1ccc(O)c(O)c1</smiles>

1<smiles>O=C(/C=C/c1ccc(O)c(O)c1)O[C@@H](Cc1ccc(O)c(O)c1)C(=O)O</smiles><smiles>O=C(/C=C/c1ccc(O)c2c1[C@@H](C(=O)O[C@H](Cc1ccc(O)c(O)c1)C(=O)O)[C@H](c1ccc(O)c(O)c1)O2)O[C@@H](Cc1ccc(O)c(O)c1)C(=O)O</smiles><smiles>O=C(/C=C/c1ccc(O)c(O)c1/C=C/c1ccc(O)c(O)c1)O[C@@H](Cc1ccc(O)c(O)c1)C(=O)O</smiles>

4<smiles>CC(C)c1cc2c(c(O)c1O)[C@]1(C(=O)O)CCCC(C)(C)[C@@H]1CC2</smiles>

5

Figure 1. Typical phenolic compounds in Salvia genus

1: Caffeic acid, 2: Rosmarinic acid, 3: Salvianolic acid B, 4: Salvianolic acid A, 5: Carnosic acid

Caffeic acid (CAA) acts as a structural unit of a variety of the phenolic compounds from the simple monomers to condensed oligomer products (Figure 1) and plays a basic role in the biochemistry of the Salvia species [5,16]. Different CAA derivatives occur as the major hydrophilic components of the Salvia species and possess a variety of biological activities $[13,16]$. It is well known that anti-oxidative activities of Salvia species are mainly due to the presence of rosmarinic acid 
(RA) (dimer of CAA) as the most abundant phenolic compound [5,16]. Salvianolic acids as derivatives of CAA are another groups of polyphenolic metabolites whose occurrence has only been approved in the Salvia genus [16-19]. Among these phenolic acids, salvianolic acid A (Sal A) a trimeric derivative of $\mathrm{CAA}$, and salvianolic acid $\mathrm{B}(\mathrm{Sal} \mathrm{B})$ a dimer derivative of RA, and thus a tetramer of CAA, are two of the most important ones [16,20,21]. It has been demonstrated that Sal A as the most effective salvianolic acid in Salvia genus has antioxidative, antitumor, biomembrane and cardiovascular protection effects [16,21]. Salvianolic acid B, as the most abundant phenolic compound of $S$. miltiorrhiza is widely used in clinical practice for the treatment of cardiovascular disorders and liver diseases in China [21, 22].

Carnosic acid (CA) is a phenolic diterpene of the abietane type compounds [23] that has received great attention as a strong antioxidant in food and biomedical sciences [24]. This phenolic diterpene is found frequently in Rosmarinus officinalis and $S$. officinalis [23,24]. The presence of CA has also been confirmed in other species of Lamiaceae including Satureja montana, S. sclarea, S. glutinosa [25], $S$. mellifera [19], S. eremophila and S. santolinifolia [26].

Since the pharmaceutical value of phenolic compounds is well known and Salvia species are widely distributed in Iran, the limited data available prompted the present investigation of the identification and determination of some phenolic compounds in some wild Salvia species from Iran.

\section{Materials and Methods}

\subsection{Plant Materials}

The 41 samples of 27 Salvia species were harvested at the flowering stage from different localities of Iran during the spring and summer 2012. The voucher specimens were deposited at the central herbarium of Bu-Ali Sina University (BASUH) and Herbarium of Biology Department, Hormozgan University (HAPH), Iran. The scientific names, collection sites and voucher numbers of the studied species are listed in Table 1.

\subsection{Chemicals and Standards}

All the analytical and HPLC-grade solvents were supplied from Merck Chemical Co. Ltd. (Darmstadt, Germany). Standards samples of carnosic acid, salvianolic acid B and caffeic acid were purchased from Sigma, rosmarinic acid and salvianolic acid A were supplied from Aldrich and Fluka, respectively.

\subsection{Phenolic Compounds Extraction from Samples}

One gram of the air-dried and finely powdered leaf and root samples of the examined Salvia species were separately extracted by maceration in methanol $(2 \times 10 \mathrm{~mL}$ for $24 \mathrm{~h})$ at room temperature and dark place. After filtration, the solvents were removed on a rotary evaporator under vacuum at $40^{\circ} \mathrm{C}$ to dryness. The crude extracts were stored in the dark at $4^{\circ} \mathrm{C}$ until analysis. The dried extracts freshly were dissolved in $99 \%$ ethanol at $4 \mathrm{mg} / \mathrm{mL}$ concentration for further studies [27].

\subsection{HPLC Analysis}

Phenolic compounds in the samples were identified and quantified using a Smartline HPLC instrument (Kenuer, Germany) equipped with a quaternary pump and a UV-VIS detector (D-14163 model). Reverse phase chromatography separation was performed with a C18 Eurospher-100 (5 $\mu \mathrm{m}$ particle, $125 \mathrm{~mm} \times 4 \mathrm{~mm}$ ). The chromatographic data were processed using ChromGate software (version 3.1). The flow rate used for column elution was $1 \mathrm{~mL} / \mathrm{min}$ and peaks were monitored by UV detection at $280 \mathrm{~nm}$. The sample injection volume was $20 \mu \mathrm{L}$. The solvent system was $0.2 \%(\mathrm{v} / \mathrm{v})$ glacial acetic acid in water (solvent A) and acetonitrile (solvent B). The gradient elution program was as follows: $90-75 \% \mathrm{~A}(\mathrm{v} / \mathrm{v})$ at $0-15 \mathrm{~min}, 75-20 \% \mathrm{~A} \mathrm{(v/v)} \mathrm{at} 15-40 \mathrm{~min}, 20-0 \% \mathrm{~A}(\mathrm{v} / \mathrm{v})$ at $40-45 \mathrm{~min}$, 
0\% A (v/v) at 45-50 min, 0-90\% A (v/v) at 50-55 min. Samples and standard solutions were filtered through $0.45 \mu \mathrm{m}$ hydrophilic PTFE membrane filters before injection. Identification of the compounds in the chromatograms was performed by comparison of their retention times with those of reference standards. Determination of the each phenolic compound was performed using the corresponding calibration curve. Extract samples were injected three times to HPLC for analysis.

Table 1. Scientific name, locality and voucher number of the studied Iranian Salvia species

\begin{tabular}{|c|c|c|c|c|c|c|}
\hline Species (population) & Location (province) & $\begin{array}{l}\text { Collection } \\
\text { Time }\end{array}$ & $\begin{array}{l}\text { Latitude } \\
\text { (N) }\end{array}$ & $\begin{array}{l}\text { Longitude } \\
\text { (E) }\end{array}$ & $\begin{array}{l}\text { Altitude } \\
\text { (m) }\end{array}$ & $\begin{array}{l}\text { Voucher } \\
\text { number }\end{array}$ \\
\hline S. aegyptiaca L. & Hormozgan- Geno mountain & April & $27^{\circ} 26^{\prime} 45^{\prime \prime}$ & $56^{\circ} 18^{\prime} 17^{\prime \prime}$ & 235 & HAPH-92138 \\
\hline S. aethiopis L. & North Khorasan- Rooieen & June & $37^{\circ} 23^{\prime} 11.66^{\prime \prime}$ & $57^{\circ} 02^{\prime} 37.32^{\prime \prime}$ & 1889 & BASU 32969 \\
\hline S. aristata Aucher ex Benth. & Qazvin- Gardane Kaman & July & $36^{\circ} 27^{\prime} 49^{\prime \prime}$ & $50^{\circ} 07^{\prime} 59^{\prime \prime}$ & 2034 & BASU 34046 \\
\hline S. atropatana Bunge. & Alborz- Kandovan & June & $36^{\circ} 10^{\prime} 20^{\prime \prime}$ & $51^{\circ} 18^{\prime} 53^{\prime \prime}$ & 2581 & BASU 34027 \\
\hline S. ceratophylla L. (T) & Tehran- Damavand & June & $35^{\circ} 40^{\prime} 56^{\prime \prime}$ & $52^{\circ} 01^{\prime} 45^{\prime \prime}$ & 2020 & BASU 34058 \\
\hline S. ceratophylla L. (M) & Razavi Khorasan- Abghad & June & $36^{\circ} 30^{\prime} 26.6^{\prime \prime}$ & $59^{\circ} 02^{\prime} 18.41^{\prime \prime}$ & 1450 & BASU 33989 \\
\hline S. choloroleuca Rech. f. \& Aell. (T1) & Tehran-Shemshak & June & $35^{\circ} 59^{\prime} 27.7^{\prime \prime}$ & $51^{\circ} 28^{\prime} 55.5^{\prime \prime}$ & 2365 & BASU 34022 \\
\hline S. choloroleuca Rech. f. \& Aell. (T2) & Tehran- Darbandsar & July & $36^{\circ} 00^{\prime} 00^{\prime \prime}$ & $51^{\circ} 29^{\prime} 00^{\prime \prime}$ & 2450 & BASU 34038 \\
\hline S. choloroleuca Rech. f. \& Aell. (M) & Razavi Khorasan- Ferizi & June & $40^{\circ} 37^{\prime} 32^{\prime \prime}$ & $40^{\circ} 67^{\prime} 45^{\prime \prime}$ & 1965 & BASU 34094 \\
\hline S. chorassanica Bunge. & Razavi Khorasan- Binalud mountain & July & $36^{\circ} 15^{\prime} 57^{\prime \prime}$ & $59^{\circ} 07^{\prime} 41.3^{\prime \prime}$ & 2790 & BASU 34047 \\
\hline S. hydrangea DC. & Qazvin- Razjerd village & July & $36^{\circ} 20^{\prime} 43^{\prime \prime}$ & $50^{\circ} 09^{\prime} 26^{\prime \prime}$ & 1609 & BASU 34510 \\
\hline S. hypoleuca Benth. (T1) & Alborz- Gajereh & July & $36^{\circ} 06^{\prime} 08^{\prime \prime}$ & $51^{\circ} 20^{\prime} 56^{\prime \prime}$ & 2335 & BASU 34037 \\
\hline S. hypoleuca Benth. (T2) & Tehran- Jajrud & July & $35^{\circ} 44^{\prime} 52^{\prime \prime}$ & $51^{\circ} 43^{\prime} 07^{\prime \prime}$ & 1570 & BASU 33080 \\
\hline S. hypoleuca Benth. (Q) & Qazvin- Khanjarbolagh village & July & $36^{\circ} 26^{\prime} 16^{\prime \prime}$ & $50^{\circ} 13^{\prime} 05^{\prime \prime}$ & 2112 & BASU 34512 \\
\hline S. leriifolia Benth. & Razavi Khorasan- Bojestan & April & $34^{\circ} 22^{\prime} 31.81^{\prime \prime}$ & $58^{\circ} 41^{\prime} 9.33^{\prime \prime}$ & 1300 & BASU 34050 \\
\hline S. limbata C.A. Mey. & Qazvin- Zereshk & July & $36^{\circ} 25^{\prime} 59.39^{\prime \prime}$ & $50^{\circ} 06^{\prime} 11.18^{\prime \prime}$ & 2000 & BASU 34511 \\
\hline S. macrosiphon Boiss. & Razavi Khorasan- Kalat road & July & $36^{\circ} 36^{\prime} 4.56^{\prime \prime}$ & $59^{\circ} 54^{\prime} 8.73^{\prime \prime}$ & 1821 & BASU 33990 \\
\hline S. mirzayanii Rech. f. \& Esfand. & Hormozgan- Tangezagh & April & $27^{\circ} 37^{\prime} 56^{\prime \prime}$ & $56^{\circ} 12^{\prime} 11^{\prime \prime}$ & 410 & НАРН-91321 \\
\hline S. nemorosa L. (T) & Tehran- Firozkoh & July & $35^{\circ} 43^{\prime} 39^{\prime \prime}$ & $52^{\circ} 40^{\prime} 23^{\prime \prime}$ & 1855 & BASU 34072 \\
\hline S. nemorosa L. (M) & Razavi Khorasan- Tangalshur & June & $36^{\circ} 14^{\prime} 17.93^{\prime \prime}$ & $59^{\circ} 40^{\prime} 25.48^{\prime \prime}$ & 973.5 & BASU 34232 \\
\hline S. nemorosa L. (Q) & Qazvin- Aliabad forest & June & $36^{\circ} 26^{\prime} 1^{\prime \prime}$ & $49^{\circ} 9^{\prime} 53^{\prime \prime}$ & 1940 & BASU 33997 \\
\hline S. $\times$ sylvestris $($ IBRC) & Iranian Biological Resources Center & July & - & - & - & IBRC P1000875 \\
\hline S. officinalis $\mathrm{L}$. & Razavi Khorasan- Tangalshur & June & $36^{\circ} 14^{\prime} 17.93^{\prime \prime}$ & $59^{\circ} 40^{\prime} 25.48^{\prime \prime}$ & 937.5 & BASU 33995 \\
\hline S. reuterana Boiss. (T1) & Tehran- Zaigan & July & $35^{\circ} 56^{\prime} 49^{\prime \prime}$ & $51^{\circ} 32^{\prime} 51^{\prime \prime}$ & 2030 & BASU 34070 \\
\hline S. reuterana Boiss. (T2) & Tehran- Damavand road & July & $35^{\circ} 42^{\prime} 21^{\prime \prime}$ & $51^{\circ} 59^{\prime} 14^{\prime \prime}$ & 2032 & BASU 33992 \\
\hline S. santolinifolia Boiss. & Hormozgan- Tangezagh & April & $27^{\circ} 36^{\prime} 54^{\prime \prime}$ & $56^{\circ} 12^{\prime} 45^{\prime \prime}$ & 398 & НАРН-91239 \\
\hline S. sclarea L. (T) & Tehran- Damavand & July & $35^{\circ} 44^{\prime} 43^{\prime \prime}$ & $52^{\circ} 03^{\prime} 39^{\prime \prime}$ & 2160 & BASU 34039 \\
\hline S. sclarea L. (M) & North Khorasan- Rooieen & July & $37^{\circ} 23^{\prime} 11.66^{\prime \prime}$ & $57^{\circ} 2^{\prime} 37.32^{\prime \prime}$ & 1889 & BASU 32965 \\
\hline S. sclarea L. (Q) & Qazvin- Joladak village & July & $36^{\circ} 22^{\prime} 19^{\prime \prime}$ & $50^{\circ} 32^{\prime} 10^{\prime \prime}$ & 2200 & BASU 34079 \\
\hline S. sharifii Rech. f. \& Esfand. & Hormozgan- Geno mountain & April & $27^{\circ} 26^{\prime} 29^{\prime \prime}$ & $56^{\circ} 18^{\prime} 11^{\prime \prime}$ & 198 & НАРН-92054 \\
\hline S. staminea Montbr. \& Auch. ex Benth. & Alborz- Kandovan & June & $36^{\circ} 10^{\prime} 20^{\prime \prime}$ & $51^{\circ} 18^{\prime} 53^{\prime \prime}$ & 2581 & BASU 34077 \\
\hline S. syriaca $\mathrm{L}$. & Qazvin- Joladak village & July & $36^{\circ} 22^{\prime} 19^{\prime \prime}$ & $50^{\circ} 32^{\prime} 10^{\prime \prime}$ & 2200 & BASU 34052 \\
\hline S. tebesana Bunge. & South Khorasan- Bandar dare & July & $32^{\circ} 48^{\prime} 59.68^{\prime \prime}$ & $59^{\circ} 12^{\prime} 58.53^{\prime \prime}$ & 1720 & BUSU 34588 \\
\hline S. verticillata $\mathrm{L} .(\mathrm{T} 1)$ & Tehran- Shemshak & June & $35^{\circ} 52^{\prime} 13^{\prime \prime}$ & $51^{\circ} 32^{\prime} 25^{\prime \prime}$ & 1826 & BASU 34023 \\
\hline S. verticillata L. (T2) & Tehran- Darbandsar & July & $36^{\circ} 00^{\prime} 23^{\prime \prime}$ & $51^{\circ} 29^{\prime} 00^{\prime \prime}$ & 2450 & BASU 34034 \\
\hline S. verticillata $\mathrm{L} .(\mathrm{Q})$ & Qazvin-Joladak village & July & $36^{\circ} 22^{\prime} 19^{\prime \prime}$ & $50^{\circ} 32^{\prime} 10^{\prime \prime}$ & 2200 & BASU 33996 \\
\hline S. verticillata L. (IBRC) & Iranian Biological Resources Center & July & $36^{\circ} 28^{\prime} 28.5^{\prime \prime}$ & $50^{\circ} 24^{\prime} 57.5^{\prime \prime}$ & 1536 & IBRC P1003833 \\
\hline S. virgata Jacq. (Q) & Qazvin- Aghagir village & June & $36^{\circ} 23^{\prime} 08^{\prime \prime}$ & $50^{\circ} 24^{\prime} 08^{\prime \prime}$ & 1405 & BASU 34513 \\
\hline S. virgata Jacq. (M) & North Khorasan- Rooieen & July & $37^{\circ} 11^{\prime} 41.61^{\prime \prime}$ & $57^{\circ} 29^{\prime} 29.75^{\prime \prime}$ & 1760 & BASU 34044 \\
\hline S. viridis $\mathrm{L}$. & Qazvin- Rajaee dasht & June & $36^{\circ} 27^{\prime} 33^{\prime \prime}$ & $50^{\circ} 17^{\prime} 01^{\prime \prime}$ & 1135 & BASU 34088 \\
\hline S. xanthocheila Boiss. ex Benth. & Qazvin- Pich bon village & July & $36^{\circ} 26^{\prime} 16^{\prime \prime}$ & $50^{\circ} 13^{\prime} 05^{\prime \prime}$ & 2112 & BASU 34041 \\
\hline
\end{tabular}

\subsection{Method Validation}

\subsubsection{Linearity}

The standard stock solutions were separately prepared in pure ethanol and diluted to appropriate concentration range for the establishment of calibration curves. The calibration curves were plotted on the basis of linear regression analysis of the integrated peak areas $(\mathrm{y})$ versus concentrations $(\mathrm{x}, \mu \mathrm{g} / \mathrm{mL})$ of the five authentic compounds at different levels (Table 2). Correlation coefficients were considered to confirm the significant linear calibration for the standard samples. 
Table 2. Validation parameters of HPLC method for the reported phenolic compounds

\begin{tabular}{|c|c|c|c|c|c|c|}
\hline Compounds & $\begin{array}{c}\text { Purity } \\
\text { percentage }\end{array}$ & $\begin{array}{c}\text { Stock concentration } \\
(\mathrm{mg} / \mathrm{mL})\end{array}$ & $\begin{array}{c}\text { Linear range } \\
(\mu \mathrm{g} / \mathrm{mL})\end{array}$ & Regression equation & $R^{2}$ & $\begin{array}{l}\text { RSD } \\
(\%)\end{array}$ \\
\hline Rosmarinic acid & $96 \%$ & 1 & $0-100$ & $y=42197 x-41350$ & 0.9965 & 0.5 \\
\hline Salvianolic acid B & $\geq 94 \%$ & 1 & $0-1000$ & $\mathrm{y}=2055.1 \mathrm{x}+54.199$ & 0.9938 & 0.4 \\
\hline Salvianolic acid A & $\geq 95 \%$ & 0.9 & $0-80$ & $y=20037 x-30584$ & 0.9976 & 0.3 \\
\hline Carnosic acid & $\geq 95 \%$ & 1 & $0-600$ & $y=4519.9 x+62174$ & 0.9683 & 1.5 \\
\hline Caffeic acid & $\geq 98 \%$ & 6 & $0-180$ & $y=7053.4 x+12476$ & 0.9979 & 0.5 \\
\hline
\end{tabular}

\subsubsection{Precision and Recovery}

The precision of the developed HPLC method was performed with different concentrations of five standard compounds. Twenty microliters of each standard solution was injected into the HPLC system continuously under the same chromatographic conditions for five times. The percent relative standard deviation (RSD\%) for the peak area of each standard were calculated.

To evaluate of the accuracy of the applied method, recovery experiments were performed after adding three varying quantities of the standards to the samples (leaves of $S$. officinalis). Recovery values of the five components were measured from the corresponding calibration curve, and RSDs were calculated.

\subsection{Statistical Analysis}

Data were reported as means \pm SE of three independent test. The means were compared using the one-way ANOVA test and multivariate analysis of variance followed by Duncan's multiple range tests $(P<0.05)$. Graphs were drawn using Excel software. Statistical analysis was conducted with SPSS (version 22.0) software.

\section{Results and Discussion}

\subsection{Optimizing of HPLC Condition}

In this survey, different HPLC parameters were tested and compared. The phenolic compounds were detected at 260,280, 290 and $330 \mathrm{~nm}$ wavelengths, tested mobile phases were consisted of acetonitrile-water system and methanol-water system and mobile phase flow rates were $0.5,0.7,0.8$, 1 and $1.2 \mathrm{~mL} / \mathrm{min}$. The binary mixtures of the acetonitrile-water system were more effective for the separation of the detected compounds. Addition of $2 \%(\mathrm{v} / \mathrm{v})$ acetic acid to water improved peaks shape and separation efficiency. Due to greater baseline stability, mobile phase system of acetonitrile- $2 \%$ aqueous acetic acid was selected. Also, the results showed that the most suitable elution flow rate was $1 \mathrm{~mL} / \mathrm{min}$ and all the examined compounds were well detected at wavelength of $280 \mathrm{~nm}$. Under these experimental conditions, all the five compounds were eluted within a run time of $60 \mathrm{~min}$ and the separation was adequate.

\subsection{Method Validation}

The method was validated by the linearity, precision and reproducibility of the results. Regression equations were derived from the external standard method. The correlation coefficient of the equations $\left(R^{2}\right)$ was over 0.9683 , which indicated all the standard compounds showed good linearity in the relatively wide concentration. After determination, precision and repeatability (RSD of intraday) of the five authentic substances was below 1.5\% (Table 2). The RSDs were taken as a measure of 
precision and their values indicated that the instrument was highly precise. The average recovery of the five components and RSDs were obtained (Table 3) and showed that the method had a good accuracy.

Table 3. A sample recovery data of the reported compounds in the leaves of S. officinalis

\begin{tabular}{cccccc}
\hline Compound & $\begin{array}{c}\text { Sample contents } \\
(\mathbf{m g} / \mathbf{g})\end{array}$ & $\begin{array}{c}\text { Added } \\
(\mathbf{m g} / \mathbf{g})\end{array}$ & $\begin{array}{c}\text { Recovery } \\
(\mathbf{\%})\end{array}$ & $\begin{array}{c}\text { Mean recovery } \\
(\mathbf{\%})\end{array}$ & $\begin{array}{c}\text { RSD } \\
(\mathbf{\%})\end{array}$ \\
\hline \multirow{2}{*}{ RA } & \multirow{2}{*}{15.02} & 7.50 & 99.84 & & \\
& & 11.30 & 100.10 & 100.08 & \\
Sal B & 7.03 & 18.00 & 100.30 & & \\
& & 3.50 & 96.87 & & \\
& & 4.75 & 97.45 & 97.16 & \\
Sal A & 4.55 & 9.20 & 97.15 & & \\
& & 2.30 & 98.20 & & \\
& & 3.35 & 97.65 & & \\
CA & 7.75 & 5.50 & 99.50 & & \\
& & 3.87 & 96.03 & & \\
CAA & & 5.40 & 95.65 & & \\
& \multirow{2}{*}{1.82} & 9.00 & 96.50 & & \\
\end{tabular}

\subsection{Quantitative Analysis of Samples}

In the present study, the presence and the amount of some phenolic compounds (RA, CA, CAA, Sal A and Sal B) were simultaneously assessed in 41 populations from 27 wild Salvia species of Iran by HPLC. Based on the literature, this is the first report about the existence of the studied phenolic acids in some Salvia species, including S. aegyptiaca, S. aristata, S. atropatana, S. ceratophylla, S. chorassanica, S. hydrangea, S. mirzayanii, S. reuterana, S. sharifii, S. tebesana, S. xanthocheila and S. syriaca. Qualitative analysis of ethanolic extracts of the studied organs by HPLC confirmed the presence of the phenolic compounds. As shown in the Figure 2, HPLC chromatograms of the extracts exhibited five main peaks with the retention times of 20.5, 21.88, 23.05, 34.5 and $37.3 \mathrm{~min}$, corresponding to RA, Sal B, Sal A, CA and CAA, respectively.

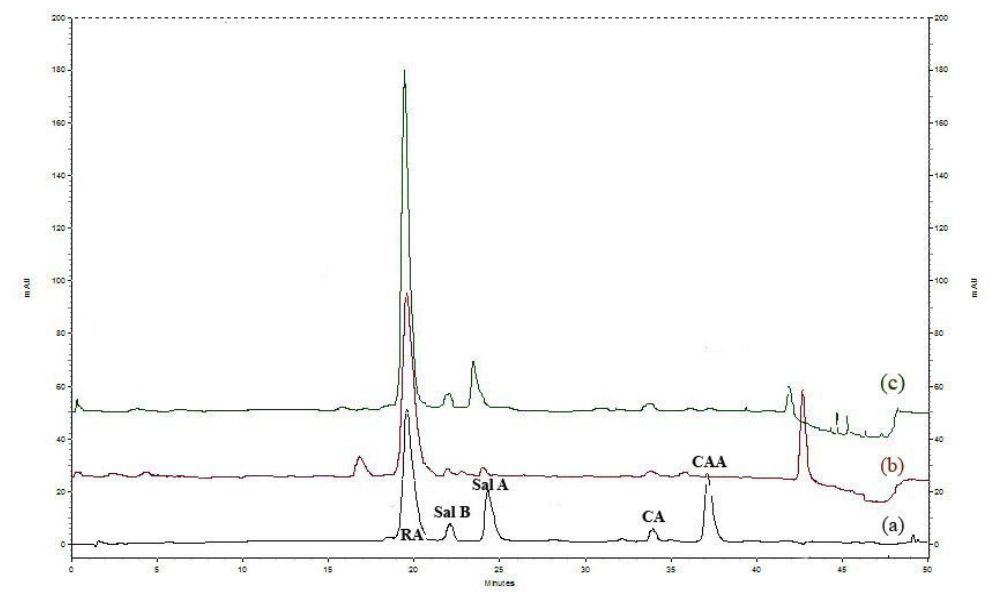

Figure 2. Representative HPLC chromatograms of the five phenolic components in the standard solution (a) and in the ethanolic extract of the roots (b) and leaves (c) of S. verticillata (IBRC);

RA: rosmarinic acid, Sal B: salvianolic acid B, Sal A: salvianolic acid A, CA: carnosic acid,

CAA: caffeic acid

Our findings showed the great variations in the contents of phenolic compounds among the studied Salvia species at both intra- and inter-species levels. The results showed that the leaves were rich in phenolic compounds with Sal B being the most abundant with descending amounts of RA, CA and Sal A in that order (Table 4). 
Table 4. The content of determined phenolic compounds in the leaves and roots of 41 populations from 27 Iranian Salvia species

\begin{tabular}{|c|c|c|c|c|c|c|c|c|c|c|c|c|}
\hline \multirow{2}{*}{$\begin{array}{l}\text { Species } \\
\text { (population) }\end{array}$} & \multicolumn{2}{|c|}{$\begin{array}{c}\text { Yield } \\
(\%)\end{array}$} & \multicolumn{2}{|c|}{$\begin{array}{c}\text { Rosmarinic acid } \\
(\mathrm{mg} / \mathrm{g} \mathrm{DW})\end{array}$} & \multicolumn{2}{|c|}{$\begin{array}{c}\text { Salvianolic acid B } \\
(\mathrm{mg} / \mathrm{g} \text { DW })\end{array}$} & \multicolumn{2}{|c|}{$\begin{array}{c}\text { Salvianolic acid A } \\
(\mathrm{mg} / \mathrm{g} \mathrm{DW})\end{array}$} & \multicolumn{2}{|c|}{$\begin{array}{c}\text { Carnosic acid } \\
(\mathrm{mg} / \mathrm{g} \text { DW) }\end{array}$} & \multicolumn{2}{|c|}{$\begin{array}{l}\text { Caffeic acid } \\
(\mathrm{mg} / \mathrm{g} \text { DW) }\end{array}$} \\
\hline & Leaf & Root & Leaf & Root & Leaf & Root & Leaf & Root & Leaf & Root & Leaf & Root \\
\hline S. aegyptiaca & 13.00 & - & $2.15 \pm 0.49^{\mathrm{p}-\mathrm{r}}$ & & $0.00 \pm 0.00^{r}$ & - & $0.67 \pm 0.10^{1-q}$ & - & $3.38 \pm 0.42^{i-\mathbf{n}}$ & & $1.74 \pm 0.05^{\mathrm{n}-\mathrm{q}}$ & - \\
\hline S. aethiopis & 15.00 & 5.40 & $4.25 \pm 1.04^{k-n}$ & $1.27 \pm 0.61^{\mathrm{k}-0}$ & $5.04 \pm 1.21^{0-q}$ & $1.78 \pm 0.16^{\mathrm{kl}}$ & $0.70 \pm 0.29^{\mathrm{k}-\mathrm{q}}$ & $0.04 \pm 0.01^{\mathrm{jk}}$ & $8.50 \pm 0.87^{\mathrm{hi}}$ & $0.00 \pm 0.00^{\mathrm{i}}$ & $7.83 \pm 0.48^{\mathrm{i}}$ & $5.65 \pm 0.38^{\mathrm{d}}$ \\
\hline S. aristata & 16.50 & 21.80 & $0.45 \pm 0.03^{\mathrm{s}}$ & $0.94 \pm 0.36^{\mathrm{n}-\mathrm{o}}$ & $6.38 \pm 0.66^{\mathrm{n}-\mathrm{p}}$ & $5.21 \pm 0.18^{\mathrm{a}}$ & $0.31 \pm 0.01^{1-q}$ & $2.45 \pm 0.14^{\mathrm{a}}$ & $1.15 \pm 0.03^{\mathrm{n}-\mathrm{p}}$ & $0.00 \pm 0.00^{\mathrm{i}}$ & $3.78 \pm 0.16^{j-o}$ & $13.29 \pm 0.64^{\mathrm{b}}$ \\
\hline S. atropatana & 19.20 & 15.00 & $6.55 \pm 0.51^{\mathrm{g}-\mathrm{i}}$ & $1.65 \pm 0.24^{\mathrm{j}-\mathrm{n}}$ & $14.76 \pm 0.44^{j \mathrm{k}}$ & $3.18 \pm 0.14^{\text {e-h }}$ & $1.55 \pm 0.53^{\mathrm{g}-\mathrm{I}}$ & $0.46 \pm 0.06^{\mathrm{e}-\mathrm{f}}$ & $18.18 \pm 2.53^{\mathrm{c}}$ & $1.47 \pm 0.06^{\mathrm{g}}$ & $8.38 \pm 0.83^{\text {hi }}$ & $1.05 \pm 0.06^{\text {f-k }}$ \\
\hline S. ceratophylla (T) & 6.80 & 9.00 & $3.34 \pm 0.17^{\mathrm{n}-\mathrm{p}}$ & $2.10 \pm 0.14^{\mathrm{h}-\mathrm{I}}$ & $2.81 \pm 0.12^{q r}$ & $3.33 \pm 0.16^{\mathrm{ef}}$ & $0.54 \pm 0.02^{\mathrm{m}-\mathrm{q}}$ & $0.47 \pm 0.12^{\mathrm{ce}}$ & $0.00 \pm 0.00^{\mathrm{p}}$ & $2.73 \pm 0.35^{\mathrm{f}}$ & $0.06 \pm 0.02^{q}$ & $5.43 \pm 0.25^{\mathrm{d}}$ \\
\hline S. ceratophylla (M) & 15.00 & 10.30 & $3.19 \pm 0.05^{\mathrm{n}-\mathrm{p}}$ & $1.17 \pm 0.10^{\mathrm{l}-\mathrm{o}}$ & $4.88 \pm 0.29^{0-q}$ & $0.09 \pm 0.01^{\mathrm{pq}}$ & $1.62 \pm 0.11^{\mathrm{g}-\mathrm{k}}$ & $0.14 \pm 0.03^{\mathrm{h}-\mathrm{k}}$ & $3.38 \pm 0.41^{i-n}$ & $2.64 \pm 0.30^{\mathrm{f}}$ & $2.10 \pm 0.21^{\mathrm{m}-\mathrm{q}}$ & $7.94 \pm 0.15^{\mathrm{c}}$ \\
\hline S. choloroleuca (T1) & 17.40 & 6.50 & $0.31 \pm 0.06^{\mathrm{rs}}$ & $1.92 \pm 0.03^{\mathrm{i}-\mathrm{m}}$ & $15.49 \pm 0.86^{\mathbf{j k}}$ & $4.20 \pm 0.12^{\mathrm{bc}}$ & $0.33 \pm 0.04^{0-q}$ & $0.16 \pm 0.02^{\mathrm{g}-\mathrm{k}}$ & $1.15 \pm 0.06^{\mathrm{n}-\mathrm{p}}$ & $2.26 \pm 0.43^{\mathrm{f}}$ & $1.94 \pm 0.57^{\mathrm{pq}}$ & $<$ LOD \\
\hline S. choloroleuca (T2) & 13.40 & 6.80 & $1.00 \pm 0.36^{\mathrm{rs}}$ & $1.99 \pm 0.11^{\mathrm{i}-\mathrm{m}}$ & $13.13 \pm 0.65^{\mathrm{kl}}$ & $3.51 \pm 0.10^{\text {de }}$ & $0.40 \pm 0.08^{\mathrm{n}-\mathrm{q}}$ & $0.19 \pm 0.05^{\mathrm{g}-\mathrm{k}}$ & $2.35 \pm 0.49^{\mathrm{m}-\mathrm{o}}$ & $0.93 \pm 0.09^{\mathrm{h}}$ & $1.02 \pm 0.12^{\mathrm{m}-\mathrm{q}}$ & $<$ LOD \\
\hline S. choloroleuca (M) & 18.00 & 5.50 & $0.92 \pm 0.46^{\mathrm{rs}}$ & $0.75 \pm 0.14^{\mathrm{n}-\mathrm{o}}$ & $8.03 \pm 0.05^{\mathrm{m}-0}$ & $4.68 \pm 0.10^{\mathbf{b}}$ & $0.82 \pm 0.22^{\mathrm{k}-\mathrm{q}}$ & $0.09 \pm 0.02^{i-k}$ & $3.98 \pm 0.33^{\mathrm{k}-\mathrm{m}}$ & $0.00 \pm 0.00^{\mathrm{i}}$ & $3.80 \pm 0.46^{\mathrm{j}-\mathrm{o}}$ & $0.91 \pm 0.03^{\mathrm{g}-\mathrm{k}}$ \\
\hline S. chorassanica & 24.30 & 11.30 & $6.09 \pm 1.10^{\mathrm{g}-\mathrm{j}}$ & $1.93 \pm 0.23^{\mathrm{i}-\mathrm{m}}$ & $26.38 \pm 0.25^{\mathrm{e}}$ & $0.58 \pm 0.05^{\mathrm{n}-\mathrm{q}}$ & $2.63 \pm 0.25^{\mathrm{ef}}$ & $0.05 \pm 0.01^{\mathbf{j k}}$ & $17.12 \pm 0.21^{\text {cd }}$ & $0.00 \pm 0.00^{\mathrm{i}}$ & $29.60 \pm 1.21^{\mathrm{c}}$ & $1.60 \pm 0.03^{e-i}$ \\
\hline S. hydrangea & 9.60 & 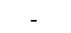 & $5.51 \pm 0.74^{\mathrm{g}-\mathrm{j}}$ & & $20.35 \pm 0.78^{\text {g-h }}$ & & $0.24 \pm 0.02^{\mathrm{pq}}$ & & $1.61 \pm 0.27^{\mathrm{n}-\mathrm{p}}$ & & $0.06 \pm 0.02^{q}$ & \\
\hline S. hypoleuca (T1) & 17.80 & 19.80 & $8.52 \pm 0.28^{\mathrm{f}}$ & $1.92 \pm 0.09^{\mathrm{i}-\mathrm{m}}$ & $30.08 \pm 1.69^{\mathrm{d}}$ & $1.22 \pm 0.11^{1-\mathrm{n}}$ & $6.79 \pm 0.91^{\mathbf{b}}$ & $0.28 \pm 0.05^{\mathrm{d}-\mathrm{j}}$ & $10.98 \pm 0.18^{\mathrm{fg}}$ & $0.00 \pm 0.00^{\mathrm{i}}$ & $12.56 \pm 1.15^{\mathrm{g}}$ & $0.32 \pm 0.06^{\mathrm{i}-\mathrm{k}}$ \\
\hline S. hypoleuca (T2) & 18.80 & 3.50 & $5.05 \pm 0.11^{\mathrm{i}-\mathrm{m}}$ & $0.91 \pm 0.18^{\mathrm{n}-\mathbf{0}}$ & $20.83 \pm 1.05^{\text {f-h }}$ & $1.28 \pm 0.23^{\mathrm{k}-\mathrm{n}}$ & $3.36 \pm 0.14^{\mathrm{de}}$ & $0.12 \pm 0.02^{\mathrm{h}-\mathrm{k}}$ & $10.49 \pm 0.29^{\mathrm{gh}}$ & $0.00 \pm 0.00^{\mathrm{i}}$ & $60.14 \pm 2.39^{\mathrm{a}}$ & $0.34 \pm 0.09^{\mathrm{i}-\mathrm{k}}$ \\
\hline S. hypoleuca (Q) & 24.30 & 11.10 & $12.48 \pm 0.67^{\mathrm{e}}$ & $1.93 \pm 0.30^{\mathrm{i}-\mathrm{m}}$ & $21.36 \pm 0.87^{\mathrm{fg}}$ & $0.19 \pm 0.02^{0-q}$ & $0.96 \pm 0.23^{\mathrm{i}-q}$ & $0.04 \pm 0.02^{\mathbf{j k}}$ & $10.29 \pm 0.70^{\mathrm{gh}}$ & $0.00 \pm 0.00^{\mathrm{i}}$ & $7.65 \pm 0.52^{\mathrm{i}}$ & $1.00 \pm 0.20^{\mathrm{f}-\mathrm{k}}$ \\
\hline S. leriifolia & 25.40 & 9.50 & $16.01 \pm 0.29^{\mathrm{d}}$ & $2.16 \pm 0.52^{\mathrm{h}-\mathrm{k}}$ & $17.74 \pm 0.73^{\mathrm{h}-\mathrm{j}}$ & $1.75 \pm 0.18^{\mathrm{kl}}$ & $4.10 \pm 0.35^{\mathrm{cd}}$ & $0.23 \pm 0.01^{e^{-k}}$ & $5.65 \pm 1.43^{\mathrm{k}}$ & $14.65 \pm 0.03^{\mathrm{b}}$ & $4.71 \pm 0.17^{-1-1}$ & $2.50 \pm 0.49^{\mathrm{e}}$ \\
\hline S. limbata $(\mathbf{Q})$ & 18.60 & - & $4.26 \pm 0.32^{\mathrm{k}-\mathrm{n}}$ & - & $19.15 \pm 0.09^{\mathrm{g}-\mathrm{i}}$ & - & $1.92 \pm 0.15^{\mathrm{f}-\mathrm{h}}$ & - & $0.00 \pm 0.00^{\mathrm{p}}$ & - & $48.07 \pm 1.77^{\mathrm{b}}$ & \\
\hline S. macrosiphon (M) & 13.10 & 17.30 & $9.21 \pm 0.06^{\mathrm{f}}$ & $1.14 \pm 0.50^{\mathrm{l}-\mathrm{o}}$ & $8.54 \pm 0.55^{\mathrm{mn}}$ & $0.19 \pm 0.02^{-\mathrm{qq}}$ & $2.11 \pm 0.28^{\mathrm{fg}}$ & $0.00 \pm 0.00^{\mathrm{k}}$ & $8.62 \pm .58^{\mathrm{hi}}$ & $0.00 \pm 0.00^{\mathrm{i}}$ & $7.83 \pm 0.92^{\mathrm{i}}$ & $7.91 \pm 0.53^{\mathrm{c}}$ \\
\hline S. mirzayanii & 22.50 & - & $4.79 \pm 0.55^{\mathrm{j}-\mathrm{n}}$ & $1.17 \pm 0.00$ & $23.86 \pm 1.65^{\text {ef }}$ & - & $2.65 \pm 0.27^{\mathrm{ef}}$ & - & $8.49 \pm 0.10^{\text {hi }}$ & 0.000 & $18.90 \pm 0.64^{\mathrm{e}}$ & - \\
\hline S. nemorosa (M) & 15.80 & - & $3.55 \pm 0.17^{\mathrm{m}-\mathrm{p}}$ & - & $7.12 \pm 0.16^{\text {n-p }}$ & - & $0.30 \pm 0.03^{0-q}$ & - & $1.66 \pm 0.08^{\mathrm{n}-\mathrm{p}}$ & - & $1.15 \pm 0.09^{\mathrm{pq}}$ & \\
\hline S. nemorosa (Q) & 13.80 & 11.50 & $7.07 \pm 0.13^{\mathrm{g}}$ & $4.92 \pm 0.14^{\mathrm{d}}$ & $15.44 \pm 2.41^{\mathbf{j k}}$ & $2.79 \pm 0.23^{\mathrm{f}-\mathrm{h}}$ & $1.94 \pm 0.25^{\text {f-h }}$ & $0.67 \pm 0.10^{\mathrm{c}}$ & $3.26 \pm 0.15^{i-\mathrm{n}}$ & $10.02 \pm 0.20^{\mathrm{c}}$ & $4.25 \pm 0.14^{\mathrm{j}-\mathrm{m}}$ & $<\mathrm{LOD}$ \\
\hline S. nemorosa (T) & 15.70 & 11.60 & $5.65 \pm 0.40^{\mathrm{g}-\mathrm{i}}$ & $7.15 \pm 0.78^{\mathbf{b}}$ & $5.07 \pm 0.54^{0-q}$ & $1.36 \pm 0.21^{\mathrm{k}-\mathrm{m}}$ & $0.36 \pm 0.03^{\mathrm{n}-\mathrm{q}}$ & $0.21 \pm 0.01^{\mathrm{g}-\mathrm{k}}$ & $1.83 \pm 0.10^{\mathrm{n}-\mathrm{p}}$ & $1.47 \pm 0.12^{\mathrm{g}}$ & $1.47 \pm 0.03^{0-q}$ & $<\mathrm{LOD}$ \\
\hline S. $\times$ sylvesteris & 16.00 & - & $2.64 \pm 0.06^{-\mathrm{oq}}$ & - & $2.17 \pm 0.09^{\mathrm{qr}}$ & - & $1.36 \pm 0.48^{\mathrm{g}-\mathrm{m}}$ & - & $0.00 \pm 0.00^{\mathrm{p}}$ & $1 .+71+12$ & $3.94 \pm 0.43^{\mathrm{j}-\mathrm{n}}$ & \\
\hline $\begin{array}{l}\text { S. officinalis } \\
\text { S }\end{array}$ & 20.00 & 6.90 & $15.02 \pm 0.53^{\mathrm{d}}$ & $2.31 \pm 0.25^{\mathrm{h}-\mathrm{j}}$ & $7.03 \pm 1.30^{\text {n-p }}$ & $1.35 \pm 0.09^{\mathrm{k}-\mathrm{m}}$ & $4.55 \pm 0.77^{\mathrm{c}}$ & $0.35 \pm 0.12^{\mathrm{d}-\mathrm{h}}$ & $7.75 \pm 0.82^{\mathrm{ij}}$ & $4.41 \pm 0.10^{\mathrm{d}}$ & $1.82 \pm 0.12^{\mathrm{n}-\mathrm{q}}$ & $31.77 \pm 1.60^{\mathrm{a}}$ \\
\hline S. reuterana $(\mathrm{T} 1)$ & 10.80 & 16.60 & $6.73 \pm 0.58^{\mathrm{gh}}$ & $3.02 \pm 0.14^{-\mathrm{eh}}$ & $19.07 \pm 0.62^{\mathrm{g}-\mathrm{i}}$ & $3.08 \pm 0.07^{\mathrm{e}-\mathrm{h}}$ & $1.02 \pm 0.09^{\text {h-p }}$ & $0.24 \pm 0.03^{\mathrm{d}-\mathrm{k}}$ & $15.92 \pm 0.94^{\text {de }}$ & $0.00 \pm 0.00^{\mathrm{i}}$ & $15.70 \pm 0.98^{\mathrm{f}}$ & $1.71 \pm 0.17^{\mathrm{e}-\mathrm{h}}$ \\
\hline S. reuterana (T2) & 11.70 & 9.60 & $2.35 \pm 0.20^{\mathrm{p}-\mathrm{r}}$ & $1.08 \pm 0.06^{\mathrm{m}-\mathrm{o}}$ & $17.05 \pm 0.55^{\mathrm{ij}}$ & $0.62 \pm 0.14^{\mathrm{n}-q}$ & $0.73 \pm 0.13^{\mathrm{k}-\mathrm{q}}$ & $0.02 \pm 0.01^{\mathrm{k}}$ & $31.96 \pm 0.27^{\mathrm{b}}$ & $1 \pm 0.06^{\text {gh }}$ & $22.11 \pm 1.22^{\mathrm{d}}$ & $0.43 \pm 0.10^{\mathrm{h}-\mathrm{k}}$ \\
\hline S. santolinifolia & 12.40 & - & $5.77 \pm 0.44^{\mathrm{g}-\mathrm{k}}$ & & $30.77 \pm 0.63^{\mathrm{d}}$ & - & $1.25 \pm 0.15^{\mathrm{g}-\mathrm{0}}$ & - & $5.03 \pm 0.56^{\mathrm{kl}}$ & & $1.12 \pm 0.02^{\mathrm{pq}}$ & - \\
\hline S. sclarea (M) & 15.50 & 12.30 & $5.43 \pm 0.12^{\mathbf{h}-\mathbf{i}}$ & $1.38 \pm 0.21^{\mathrm{j}-0}$ & $14.85 \pm 2.34^{j \mathbf{k}}$ & $0.00 \pm 0.00^{q}$ & $1.09 \pm 0.12^{\mathbf{h}-\mathbf{p}}$ & $0.28 \pm 0.13^{\mathrm{d}-\mathrm{j}}$ & $4.31 \pm 0.79^{\mathrm{k}-\mathrm{m}}$ & $0.00 \pm 0.00^{\mathrm{i}}$ & $5.31 \pm 0.46^{\mathrm{j}}$ & $0.67 \pm 0.19^{-k-k}$ \\
\hline S. sclarea (Q) & 19.80 & 20.50 & $4.10 \pm 0.06^{1-0}$ & $1.36 \pm 0.04^{\mathrm{j}-\mathrm{o}}$ & $50.92 \pm 2.26^{\mathbf{b}}$ & $0.75 \pm 0.46^{\mathrm{m}-\mathrm{p}}$ & $1.89 \pm 0.12^{\mathrm{f}-\mathrm{i}}$ & $0.22 \pm 0.06^{\mathrm{f}-\mathrm{k}}$ & $15.05 \pm 0.05^{\mathrm{e}}$ & $0.00 \pm 0.00^{\mathrm{i}}$ & $10.12 \pm 0.65^{\mathrm{h}}$ & $1.25 \pm 0.50^{\mathrm{e}-\mathrm{k}}$ \\
\hline S. sclarea (T) & 13.60 & 10.50 & $5.18 \pm 0.05^{\mathrm{h}-\mathrm{i}}$ & $1.40 \pm 0.16^{\mathrm{j}-\mathrm{o}}$ & $21.13 \pm 1.23^{\mathrm{fg}}$ & $0.84 \pm 0.08^{\mathrm{m}-\mathrm{o}}$ & $0.22 \pm 0.05^{\mathrm{pq}}$ & $0.10 \pm 0.05^{\mathrm{i}-\mathrm{k}}$ & $5.62 \pm 0.17^{\mathrm{k}}$ & $0.00 \pm 0.00^{\mathrm{i}}$ & $1.31 \pm 0.28^{\mathrm{pq}}$ & $1.95 \pm 0.03^{\mathrm{eg}}$ \\
\hline S. sharifii & 13.50 & - & $5.74 \pm 0.33^{\mathrm{g}-\mathrm{i}}$ & - & $6.77 \pm 0.27^{\mathrm{n}-\mathrm{p}}$ & - & $1.07 \pm 0.04^{\mathrm{h}-\mathrm{p}}$ & - & $34.05 \pm 1.18^{\mathrm{a}}$ & - & $19.37 \pm 0.65^{\mathrm{e}}$ & - \\
\hline S. staminea & 13.40 & 5.80 & $1.54 \pm 0.27^{-\mathrm{s}}$ & $1.15 \pm 0.09^{\mathbf{l}-\mathbf{0}}$ & $4.84 \pm 0.31^{0-q}$ & $1.70 \pm 0.16^{\mathrm{kl}}$ & $0.18 \pm 0.02^{\mathbf{p q}}$ & $0.16 \pm 0.08^{g-k}$ & $0.39 \pm 0.03^{\mathrm{op}}$ & $3.38 \pm 0.43^{\mathrm{e}}$ & $2.19 \pm 0.47^{\mathrm{m}-\mathrm{q}}$ & $1.68 \pm 0.18^{\mathrm{e}-\mathrm{h}}$ \\
\hline S. syriaca $(Q)$ & 14.90 & 13.90 & $5.17 \pm 0.46^{\text {h-i }}$ & $2.68 \pm 0.18^{\mathrm{j}-\mathrm{o}}$ & $54.47 \pm 2.00^{\mathrm{a}}$ & $2.60 \pm 0.27^{-\mathrm{i}}$ & $1.20 \pm 0.19^{\mathrm{g}-\mathrm{o}}$ & $0.30 \pm 0.12^{\mathrm{d}-\mathrm{i}}$ & $12.66 \pm 1.35^{\mathrm{f}}$ & $3.80 \pm 0.28^{\mathrm{e}}$ & $4.69 \pm 0.33^{5-1}$ & $2.27 \pm 0.61^{\mathrm{ef}}$ \\
\hline S. tebesana & 16.80 & 2.80 & $9.05 \pm 0.59^{\mathrm{f}}$ & $0.62 \pm 0.13^{\circ}$ & $35.04 \pm 1.76^{\mathrm{c}}$ & $1.87 \pm 0.0 \mathrm{j}^{\mathrm{j}-\mathrm{I}}$ & $1.28 \pm 0.13^{\mathrm{g}-\mathrm{n}}$ & $0.23 \pm 0.05^{-\mathrm{f}} \mathrm{k}$ & $5.69 \pm 0.40^{\mathrm{k}}$ & $0.00 \pm 0.00^{\mathrm{i}}$ & $2.93 \pm 0.25^{\mathrm{k}-\mathrm{p}}$ & $0.03 \pm 0.00^{\mathrm{k}}$ \\
\hline S. verticillata (T1) & 18.80 & 19.50 & $22.40 \pm 0.40^{\mathbf{b}}$ & $11.56 \pm 0.35^{\mathrm{a}}$ & $0.80 \pm 0.02^{\mathrm{r}}$ & $4.05 \pm 0.55^{\mathbf{b}-\mathbf{d}}$ & $0.04 \pm 0.00^{\mathrm{q}}$ & $1.25 \pm 0.14^{\mathrm{b}}$ & $0.10 \pm 0.02^{\mathrm{p}}$ & $2.27 \pm 0.13^{\mathrm{f}}$ & $0.07 \pm .02^{4}$ & $1.59 \pm .32^{\mathrm{e}-\mathrm{j}}$ \\
\hline S. verticillata (T2) & 23.70 & 8.20 & $41.07 \pm 0.80^{\mathrm{a}}$ & $3.89 \pm 0.33^{\mathrm{e}}$ & $6.74 \pm 0.29^{\mathrm{n}-\mathrm{p}}$ & $1.63 \pm 0.15^{\mathrm{kl}}$ & $3.03 \pm 0.02^{\mathrm{e}}$ & $0.11 \pm 0.01^{\mathrm{h}-\mathrm{k}}$ & $1.73 \pm 0.13^{\mathrm{n}-\mathrm{p}}$ & $0.00 \pm 0.00^{\mathrm{i}}$ & $2.44 \pm 0.13^{\mathrm{i}-\mathrm{q}}$ & $0.04 \pm 0.01^{\mathrm{k}}$ \\
\hline S. verticillata (Q) & 19.40 & 15.20 & $41.53 \pm 0.88^{\mathrm{a}}$ & $5.99 \pm 0.19^{\mathrm{c}}$ & $10.91 \pm 0.35^{\mathrm{Im}}$ & $2.50 \pm 0.23^{\mathrm{h}-\mathrm{j}}$ & $1.60 \pm 0.15^{\mathrm{g}-\mathrm{I}}$ & $0.07 \pm 0.03^{i-k}$ & $4.58 \pm 0.43^{k-1}$ & $0.00 \pm 0.00^{\mathrm{i}}$ & $5.17 \pm 0.25^{\mathrm{jk}}$ & $0.28 \pm 0.13^{\mathrm{jk}}$ \\
\hline S. verticillata (IBRC) & 20.20 & 17.40 & $18.85 \pm 0.15^{\mathbf{c}}$ & $3.79 \pm 0.17^{\mathrm{ef}}$ & $4.36 \pm 0.21^{\mathrm{pq}}$ & $3.28 \pm 0.47^{\mathrm{e}-\mathrm{g}}$ & $8.10 \pm 0.35^{\mathrm{a}}$ & $0.39 \pm 0.03^{\mathrm{d}-\mathrm{g}}$ & $6.04 \pm 0.76^{\mathrm{jk}}$ & $0.21 \pm 0.12^{\mathrm{i}}$ & $3.20 \pm 0.58^{\mathrm{g}-\mathrm{p}}$ & $0.52 \pm 0.16^{\mathrm{h}-\mathrm{k}}$ \\
\hline S. virgata (M) & 17.40 & 7.80 & $5.15 \pm 0.66^{\mathrm{h}-\mathrm{m}}$ & $2.95 \pm 0.23^{\text {f-h }}$ & $8.72 \pm 0.42^{\mathrm{mn}}$ & $1.44 \pm 0.12^{\mathrm{k}-\mathrm{m}}$ & $0.44 \pm 0.03^{\mathrm{m}-\mathrm{q}}$ & $0.15 \pm 0.06^{\mathrm{g}-\mathrm{k}}$ & $1.54 \pm 0.27^{\mathrm{n}-\mathrm{p}}$ & $3.37 \pm 0.21^{\mathrm{e}}$ & $2.06 \pm 0.15^{\mathrm{m}-\mathrm{q}}$ & $0.18 \pm 0.00^{\mathrm{k}}$ \\
\hline S. virgata (Q) & 15.90 & 10.00 & $11.41 \pm 0.84^{\mathrm{e}}$ & $3.62 \pm 0.20^{\mathrm{eg}}$ & $20.77 \pm 0.56^{\text {f-h }}$ & $3.66 \pm 0.34^{\mathrm{c}-\mathrm{e}}$ & $1.80 \pm 0.29^{\mathrm{f}-\mathrm{j}}$ & $0.47 \pm 0.12^{\mathrm{cd}}$ & $5.94 \pm 0.65^{\mathbf{j k}}$ & $0.00 \pm 0.00^{\mathrm{i}}$ & $4.93 \pm 0.04^{\mathbf{j k}}$ & $1.13 \pm 0.13^{\text {f-k }}$ \\
\hline $\begin{array}{l}\text { S. viridis } \\
\end{array}$ & 20.90 & - & $1.15 \pm 0.61^{1-\mathrm{s}}$ & $\begin{array}{l}3.020 .20 \\
-\end{array}$ & $21.50 \pm 0.42^{\mathrm{fg}}$ & $\begin{array}{c}3.000 .04 \\
-\end{array}$ & $0.91 \pm 0.06^{\mathrm{j}-q}$ & - & $0.00 \pm 0.00^{\mathrm{p}}$ & - & $3.20 \pm 0.18^{\mathrm{j}-\mathrm{p}}$ & - \\
\hline S. xanthocheila & 15.30 & 13.40 & $4.65 \pm 0.45^{\mathrm{j}-\mathrm{n}}$ & $2.81 \pm 0.21^{\mathrm{g}-\mathrm{i}}$ & $4.85 \pm 0.18^{0-q}$ & $1.99 \pm 0.40^{\mathrm{i}-\mathrm{k}}$ & $2.13 \pm 0.41^{\mathrm{fg}}$ & $0.39 \pm 0.06^{\mathrm{d}-\mathrm{g}}$ & $0.00 \pm 0.00^{\mathrm{p}}$ & $18.51 \pm 0.29^{\mathrm{a}}$ & $3.80 \pm 0.12^{\mathrm{j}-\mathrm{o}}$ & $13.36 \pm 0.44^{\mathrm{b}}$ \\
\hline Total & 37.43 & 11.33 & $7.93 \pm 0.81$ & $2.61 \pm 0.23$ & $15.35 \pm 1.11$ & $2.08 \pm 0.15$ & $1.66 \pm 0.16$ & $0.32 \pm 0.05$ & $6.84 \pm 0.70$ & $2.29 \pm 0.44$ & $8.35 \pm 1.12$ & $3.34 \pm 0.64$ \\
\hline
\end{tabular}

Data are expressed as mean \pm SE. Mean values within the same column sharing a common letter are not significantly different $(P<0.05)$.

LOD: $(\leq 0.001 \mathrm{mg} / \mathrm{g} \mathrm{DW})$ 
Rosmarinic acid was detected in all of the investigated Salvia species. Based on the results, the leaves of four populations of $S$. verticillata were rich in RA, with the highest amount obtained for the leaves of $S$. verticillata $(41.53 \mathrm{mg} / \mathrm{g}$ DW) from Qazvin location. Among the studied species, leaves of S. aristata and S. choloroleuca had the lowest contents of RA $(\leq 1 \mathrm{mg} / \mathrm{g} \mathrm{DW})$.

The highest content of RA with the value of $11.56 \mathrm{mg} / \mathrm{g}$ DW was expressed in the roots of $S$. verticillata (T1), while the lowest content of this phenolic acid was found in the roots of S. tebesana $(0.62 \mathrm{mg} / \mathrm{g} \mathrm{DW})$.

The presence of RA in the members of the genus Salvia is well known [28-31]. Previous reports indicated that the RA content of the aerial parts and leaves of S. officinalis ranged from 5.5 to 39.3 $\mathrm{mg} / \mathrm{g}$ DW depending on the extraction method and collection site [13,28,32-36]. In the present study, we obtained a value of $15.02 \mathrm{mg} / \mathrm{g}$ DW for RA in the leaves of this species. In recent years, the presence of RA has been investigated in the other Salvia species [26,28,29, 32,34,37-42]. Rosmarinic acid was found in 39 wild growing populations of 35 Salvia species in China, ranging from trace amounts to $26.31 \mathrm{mg} / \mathrm{g}$ DW in the roots of $S$. maximowicziana [13]. As far as we know, the highest RA contents have been reported in the leaves of $S$. glutinosa $(47.3 \mathrm{mg} / \mathrm{g} \mathrm{DW})$ and $S$. sclarea (41.1 $\mathrm{mg} / \mathrm{g}$ DW) from Botanical Garden in Lithuania [32]. According to the results of Zengin et al. [43], aerial parts of $S$. verticillata subsp. amasiaca of Turkey were rich in RA $(67 \pm 2 \mathrm{mg} / \mathrm{g} \mathrm{DW})$. Similarly, among the studied species in this research, leaves of three populations (Q, T2 and T1) of $S$. verticillata with the values of $41.53,41.07$ and $22.40 \mathrm{mg} / \mathrm{g} \mathrm{DW}$ were rich in RA, respectively. Also, the highest content of RA $(11.56 \mathrm{mg} / \mathrm{g} \mathrm{DW})$ in the roots was reported for S. verticillata.

The content of Sal B, as the most abundant phenolic compound, varied from $0.80 \mathrm{mg} / \mathrm{g}$ DW in the leaves of $S$. verticillata (T1) to $54.47 \mathrm{mg} / \mathrm{g}$ DW in the leaves of $S$. syriaca. Salvianolic acid B was not detected in the leaf extract of S. aegyptiaca. The amount of this phenolic acid ranged from 0.09 to $5.21 \mathrm{mg} / \mathrm{g} \mathrm{DW}$ in the roots of $S$. ceratophylla (M) and $S$. aristata, respectively, although this compound was not identified in the root of $S$. sclarea (M). The amount of Sal A ranged from 0.18 $\mathrm{mg} / \mathrm{g}$ DW in the leaves of $S$. staminea to $8.10 \mathrm{mg} / \mathrm{g}$ DW in the leaves of $S$. verticillata (IBRC). On the other hand, except for the roots of $S$. aristata $(2.45 \mathrm{mg} / \mathrm{g} \mathrm{DW})$ and $S$. verticillata (T1) $(1.25 \mathrm{mg} / \mathrm{g}$ DW), Sal A content in the roots of the other species was less than $1 \mathrm{mg} / \mathrm{g} \mathrm{DW}$.

There are a few reports about the presence of salvianolic acids in Salvia species, but these are mostly limited to S. miltiorrhiza [16, 19, 44, 45]. According to the results of Min-hui et al. [13], the content of Sal B varied from trace amounts to $82.52 \mathrm{mg} / \mathrm{g}$ DW in the roots of S. bowleyana; while only trace amounts of Sal A were determined in the S. miltiorrhiza root samples. Unlike their results, we identified the presence of Sal A and Sal B in the leaf and root extracts of S. officinalis. The highest amounts for the Sal B have been reported for the roots of S. miltiorrhiza as $130.00 \mathrm{mg} / \mathrm{g} \mathrm{DW}$ [46]. Among the examined Iranian Salvia species in this research, the leaves of S. syriaca with the value of $54.47 \mathrm{mg} / \mathrm{g} \mathrm{DW}$ were the richest source of Sal B. According to our results, the content of Sal B in the leaves $(17.74 \mathrm{mg} / \mathrm{g} \mathrm{DW})$ and roots $(1.75 \mathrm{mg} / \mathrm{g} \mathrm{DW})$ of a wild population of $S$. leriifolia were much more higher than those values reported by Modarres et al. [38] in the leaves $(0.13 \mathrm{mg} / \mathrm{g} \mathrm{DW})$ and roots $(0.11 \mathrm{mg} / \mathrm{g} \mathrm{DW})$ of another wild population of the same species. In accordance with the Chinese Pharmacopoeia (2010), Sal B is an index and chemical marker for the quality control of Danshen, dried roots of $S$. miltiorrhiza, and its content should not be less than $30 \mathrm{mg} / \mathrm{g} \mathrm{DW} \mathrm{(3 \% )} \mathrm{[47].} \mathrm{Based} \mathrm{on}$ the extraction solvent, the highest amount for Sal A has been reported as $0.41 \mathrm{mg} / \mathrm{g} \mathrm{DW}$ in the roots of commercially available S. miltiorrhiza plants [48]. Inconsistent with Min-hui et al. [13] results, Sal A was also found in all of the investigated Salvia species of Iran, except for the roots of S. macrosiphon. Furthermore, in our study the maximum value of Sal A $(8.10 \mathrm{mg} / \mathrm{g} \mathrm{DW})$ was obtained in the leaves of $S$. verticillata (IBRC), which was approximately twenty times higher than that previously reported for the commercial roots of S. miltiorrhiza by Ho et al. [48].

Among the tested samples, the content of CA ranged from trace amounts to $34.05 \mathrm{mg} / \mathrm{g} \mathrm{DW}$ in the leaves of $S$. sharifii. Also, results of the content assessment of this abietane compound in 32 populations of 19 species of Salvia showed that most of the studied species did not contain this phenolic compound in their roots. However, the roots of $S$. xanthocheila, S. leriifolia and S. nemorosa (Q) with the values of $18.51,14.65$ and $10.02 \mathrm{mg} / \mathrm{g}$ DW, respectively, were the richest sources of CA. 
Most of the previous reports have been focused on the production of CA in calli, suspension cultures and shoot cultures of $R$. officinalis and S. officinalis [23, 33, 49]. According to the published reports, CA is highly unstable and its content might be changed depending on some factors such as environmental growing conditions, season of harvest $[25,50,51]$, extraction solvent and extraction methods [49, 52-54]. The contents of CA in the leaves of S. officinalis and S. sclarea in this study were significantly greater than those found in the aerial parts of the same species native to Slovenia and Croatia [25]. In an investigation on S. eremophila and S. santolinifolia from Iran, the highest contents of CA in the aerial parts have been reported as 39.05 and $9.35 \mathrm{mg} / \mathrm{g} \mathrm{DW}$, respectively [26]. Among the studied Salvia species in this survey, the leaves of $S$. sharifii $(34.05 \mathrm{mg} / \mathrm{g} \mathrm{DW})$ and S. reuterana (T2) $(31.96 \mathrm{mg} / \mathrm{g} \mathrm{DW})$ and the roots S. xanthocheila $(18.51 \mathrm{mg} / \mathrm{g} \mathrm{DW})$ and S. leriifolia $(14.65 \mathrm{mg} / \mathrm{g} \mathrm{DW})$ could be considered as the richest natural sources of CA. As far as we know, this is the first report on the presence of this valuable abietanic compound in the roots of plant species.

The CAA content of the leaves varied from $0.06 \mathrm{mg} / \mathrm{g}$ DW in $S$. ceratophylla (T) and S. hydrangea to $60.14 \mathrm{mg} / \mathrm{g}$ DW in S. hypoleuca (T2). As compared to the leaves, the amount of this phenolic acid was relatively low in the roots of Salvia species, with the highest value of $31.77 \mathrm{mg} / \mathrm{g}$ DW in the $S$. officinalis.

\subsection{PCA Analysis}

The PCA assay was performed to determine the importance of the chemical variables for discrimination and screening of the examined Salvia species. The quantitative data obtained for the five compounds examined were subjected to PCA to describe the differences in the distribution of them across various geographic locations through 41 populations from 27 Salvia species. The PCA results revealed that three principal components with eigenvalues greater than 1 explained $57.26 \%$ of the total variance (Table 5).

The PCA scores plots based on the two principal components are shown in Figure 3. The situation of each Salvia species on the scores plots can be explained with reference to the loadings plots for PC1 and PC2. The first axis (PC1), representing $27.45 \%$ of the total variance, and mainly correlated positively to Sal B (loading, 0.61), Sal A (loading, 0.60) and RA (loading, 0.59) in the roots, but negatively correlated with CA (loading, -0.76), CAA (loading, -0.65) and Sal B (loading, 0.61 ) in the leaves. The second axis (PC2) accounted for $15.53 \%$ of the total variation and showed a high positive correlation with the RA (loading, 0.86) in the leaves. Also, the PCA results clearly showed the absence of marked variances among populations of a species. On the one hand, the PC1 separated S. aristata, which was characterized by the highest content of Sal B in the roots and very low concentrations of RA in the both of leaves and roots, to the remaining species. Moreover, the PC2 could easily distinguish the leaves and roots of different populations of $S$. verticillata rich in RA. Other species, which were distributed in a larger region, based on the concentrations of phenolic compounds and their position on PC1 and PC2 were separated as the third group. The PCA results reported by Ben Farhat et al. [55], revealed that the studied Salvia species characterized based on the quantitative distribution of nine phenolic compounds in their leaves. Similarly, PCA plots confirmed that antioxidant capacities of $S$. officinalis plants, which were collected from four wild locations in Croatia, were significantly correlated with the content of polyphenols, especially RA in their leaves [36]. Liang et al. [56] based on PCA analysis of HPLC and HPLC-MS ${ }^{n}$ fingerprints data of forty-nine compounds from twenty-five root samples of $S$. miltiorrhiza which were harvested from various geographical areas in China and were processed with different methods, classified and screened the examined samples into three groups with different contents of phenolic acids and tanshinones. In a similar PCA analysis, chemical profiles of phenolic acids and tanshinones were used as suitable markers for pharmacological evaluation of wild-grown S. miltiorrhiza plants in China [57]. 
Table 5. Principal components data based on the reported phenolic compounds of Salvia species

\begin{tabular}{lccc}
\hline Compounds & \multicolumn{3}{c}{ Component } \\
\cline { 2 - 4 } & PC1 & PC2 & PC3 \\
\hline RA-L & 0.281 & $\mathbf{0 . 8 6 2}$ & 0.005 \\
RA-R & $\mathbf{0 . 5 8 7}$ & 0.538 & -0.284 \\
Sal B-L & $\mathbf{0 . 6 0 8}$ & -0.049 & -0.209 \\
Sal B-R & $\mathbf{0 . 6 1 4}$ & -0.206 & -0.391 \\
Sal A-L & -0.164 & 0.500 & 0.501 \\
Sal A-R & $\mathbf{0 . 5 9 8}$ & -0.364 & -0.180 \\
CA-L & $\mathbf{- 0 . 7 6 5}$ & 0.018 & -0.081 \\
CA-R & 0.321 & -0.091 & $\mathbf{0 . 5 9 4}$ \\
CAA-L & $\mathbf{- 0 . 6 5 5}$ & 0.065 & -0.037 \\
CAA-R & 0.270 & -0.283 & $\mathbf{0 . 7 1 1}$ \\
Eigenvalue & 2.745 & 1.553 & 1.428 \\
\%o of variance & 27.451 & 15.526 & 14.284 \\
Cumulative\% & 27.451 & 42.977 & 57.261 \\
\hline
\end{tabular}

The values higher than 0.55 are presented as bold significant.

L: leaf; R: root.

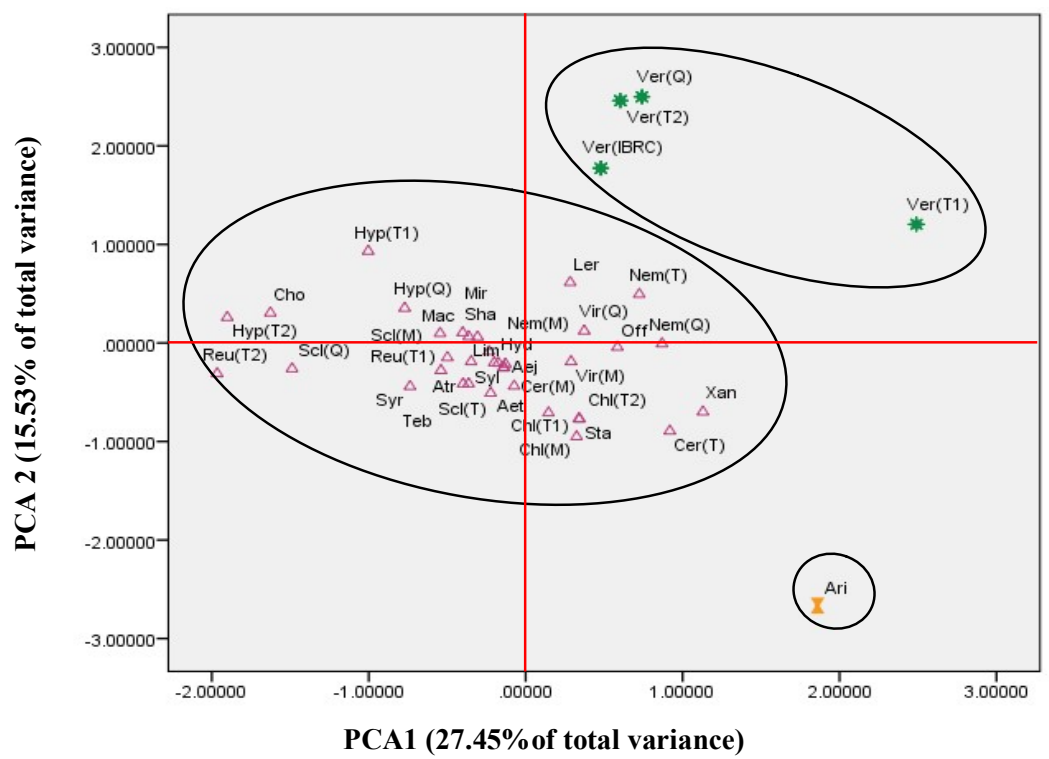

Figure 3. Scores plots of PC1 and PC2 of the PCA results obtained from distribution of 5 phenolic compounds in the leaves and roots of 41 populations from 27 Salvia species. Aeg: S. aegyptiaca, Aet: S. aethiopis, Ari: S. aristata, Atr: S. atropatana, Cer: S. ceratophylla, Chl: S. choloroleuca, Cho: S. chorassanica, Hyd: S. hydrangea, Hyp: S. hypoleuca, Ler: S. leriifolia, Lim: S. limbata, Mac: S. macrosiphon, Mir: S. mirzayanii, Nem: S. nemorosa, Off: S. officinalis, Reu: S. reuterana, San: S. santolinifolia, Scl: S. sclarea, Sha: S. sharifii, Sta: S. staminea, Syl: S. × sylvesteris, Syr: S. syriaca, Teb: S. tebesana, Ver: S. verticillata, Vir: S. virgata, Vi: S. viridis, Xan: S. xanthocheila

In Conclusion, according to our research significant differences were observed in the distribution patterns of phenolic compounds of the studied Salvia species at both of intra- and inter-species levels. It seems that the variations in contents may be due to the environmental conditions and genetic factors. Based on the collective results from phytochemical studies, S. verticillata, S. hypoleuca, $S$. leriifolia and $S$. virgata were particularly rich in rosmarinic acid. The leaves of $S$. hypoleuca, $S$. sclarea, S. tebesana, S. santolinifolia and S. syriaca had valuable amounts of salvianolic acid B. The higher amounts of salvianolic A were achieved in the leaves of $S$. hypoleuca and S. verticillata. The leaves of $S$. sharifii and $S$. reuterana had high capacity for biosynthesis and storage of carnosic acid. It 
was noticeable that plant samples of $S$. verticillata, S. hypoleuca, S. virgata and S. sclarea which were harvested from Qazvin province possessed the highest contents of phenolic compounds, as compared to the other studied populations. Due to the widespread distribution of the wild Salvia species in Iran and also according to the findings of this research and similar data from the literature, some of these species might be used as new resources of the studied compounds, especially RA, with a wide range of strong biological activities in food and medicinal industries. In general, the results of this research approved that the mentioned species can be used as potent natural sources of the studied valuable phenolic compounds for pharmaceutical, industrial, breeding, domestication and cultivation purposes, beside the $S$. officinalis which is currently known as the most important medicinal plant of the Salvia genus.

\section{Acknowledgments}

The authors are grateful to the Research Council of Shahed University for financial support during this research.

\section{ORCID}

Marzieh Fotovvat: 0000-0003-3081-4868

Tayebeh Radjabian: 0000-0001-8280-9409

Azra Saboora: 0000-0003-1691-0511

\section{References}

[1] A.H. Ebrahimabadi, A. Mazoochi, F.J. Kashi, Z. Djafari-Bidgoli and H. Batooli (2010). Essential oil composition and antioxidant and antimicrobial properties of the aerial parts of Salvia eremophila Boiss. from Iran, Food Chem. Toxicol. 48, 1371-1376.

[2] V.A. Mozaffarian 1996. Dictionary of Iranian plant names, Farhang-i Muāṣir, Tehran.

[3] Z. Sepehry Javan, F. Rahmani and R. Heidari (2012). Assessment of genetic variation of genus Salvia by RAPD and ISSR markers, Aust. J. Crop Sci. 6, 1068-1073.

[4] A.C. Gören, T. Kiliç, T. Dirmenci and G. Bilsel (2006). Chemotaxonomic evaluation of Turkish species of Salvia: Fatty acid compositions of seed oils, Biochem. Syst. Ecol. 34, 160-164.

[5] G. Kamatou, N. Makunga, W. Ramogola and A. Viljoen (2008). South African Salvia species: A review of biological activities and phytochemistry, J. Ethnopharmacol. 119, 664-672.

[6] M. Kelen and B. Tepe (2008). Chemical composition, antioxidant and antimicrobial properties of the essential oils of three Salvia species from Turkish flora, Bioresour. Technol. 99, 4096-4104.

[7] A.P. Rauter, C. Dias, A. Martins, I. Branco, N.R. Neng, J.M. Nogueira, M. Goulart, F.V. Silva, J. Justino and C. Trevitt (2012). Non-toxic Salvia sclareoides Brot. extracts as a source of functional food ingredients: Phenolic profile, antioxidant activity and prion binding properties, Food Chem. 132, 19301935.

[8] F. Naghibi, M. Mosaddegh, M. Mohammadi Motamed and A. Ghorbani (2005). Labiatae family in folk medicine in Iran: from ethnobotany to pharmacology, Iran J. Pharm. Res. 4, 63-79.

[9] M. Hamidpour, R. Hamidpour, S. Hamidpour and M. Shahlari (2014). Chemistry, pharmacology, and medicinal property of sage (Salvia) to prevent and cure illnesses such as obesity, diabetes, depression, dementia, lupus, autism, heart disease and cancer, J. Tradit. Complement. Med. 4, 82-88.

[10] I. Orhan, M. Kartal, Q. Naz, A. Ejaz, G. Yilmaz, Y. Kan, B. Konuklugil, B. Şener and M. Iqbal Choudhary (2007). Antioxidant and anticholinesterase evaluation of selected Turkish Salvia species, Food Chem. 103, 1247-1254.

[11] I. Bettaieb, N. Zakhama, W.A. Wannes, M. Kchouk and B. Marzouk (2009). Water deficit effects on Salvia officinalis fatty acids and essential oils composition, Sci. Horticult. 120, 271-275.

[12] S. Başkan, N. Öztekin and F.B. Erim (2007). Determination of carnosic acid and rosmarinic acid in sage by capillary electrophoresis, Food Chem. 101, 1748-1752.

[13] L. Min-hui, C. Jian-min, P. Yong and X. Pei-gen (2008). Distribution of phenolic acids in Chinese Salvia plants, World Sci. Technol. 10, 46-52.

[14] D. Wang, Y. Song, Y. Chen, W. Yao, Z. Li, W. Liu, S. Yue and Z. Wang (2013). Metabolic pools of phenolic acids in Salvia miltiorrhiza are enhanced by co-expression of Antirrhinum majus Delila and Roseal transcription factors, Biochem. Eng. J. 74, 115-120. 
[15] S. Munné-Bosch and L. Alegre (2001). Subcellular compartmentation of the diterpene carnosic acid and its derivatives in the leaves of rosemary, Plant Physiol. 125, 1094-1102.

[16] Y. Lu and L.Y. Foo (2002). Polyphenolics of Salvia - a review, Phytochemistry 59, 117-140.

[17] Z.-F. Zhang, H.S. Chen and Z.R. Li (2010). Simultaneous determination of five bioactive phenolic acids in Salvia yunnanensis and Salvia miltiorrhiza by HPLC, J. Chin. Pharm. Sci. 19, 271-278.

[18] B.-Q. Wang (2010). Salvia miltiorrhiza: Chemical and pharmacological review of a medicinal plant, $J$. Med. Plants Res. 4, 2813-2820.

[19] Y.-B. Wu, Z.-Y. Ni, Q.-W. Shi, M. Dong, H. Kiyota, Y.-C. Gu and B. Cong (2012). Constituents from Salvia species and their biological activities, Chem. Rev. 112, 5967-6026.

[20] J. Ho and C.-Y. Hong (2011). Salvianolic acids: small compounds with multiple mechanisms for cardiovascular protection, J. Biomed. Sci. 18, 30.

[21] H. Xia, L. Sun, H. Lou and M.M. Rahman (2014). Conversion of salvianolic acid B into salvianolic acid A in tissues of Radix Salviae miltiorrhizae using high temperature, high pressure and high humidity, Phytomedicine 21, 906-911.

[22] S.J. Zhao, J.J. Zhang, L. Yang, Z.-T. Wang and Z.-B. Hu (2011). Determination and biosynthesis of multiple salvianolic acids in hairy roots of Salvia miltiorrhiza, Acta Pharm. Sin. 46, 1352-1356.

[23] A. Kuhlmann and C. Röhl (2006). Phenolic antioxidant compounds produced by in vitro. Cultures of rosemary (Rosmarinus officinalis) and their anti-inflammatory effect on lipopolysaccharide-activated microglia, Pharm. Biol. 44, 401-410.

[24] S. Munné-Bosch and L. Alegre (2003). Drought-induced changes in the redox state of $\alpha$-tocopherol, ascorbate, and the diterpene carnosic acid in chloroplasts of Labiatae species differing in carnosic acid contents, Plant Physiol. 131, 1816-1825.

[25] M.I. Razboršek, D.B. Vončina, V. Doleček and E. Vončina (2007). Determination of Major Phenolic Acids, Phenolic Diterpenes and Triterpenes in Rosemary (Rosmarinus officinalis L.) by Gas Chromatography and Mass Spectrometry, Acta Chim. Slov. 54, 60-67.

[26] A. Jassbi, R. Miri, M. Alizadeh, M. Asadollahi, M. Massrorbabanari and I. Baldwin (2014). Quantification of Phenolic Diterpenoids and Rosmarinic Acid in Salvia eremophila and Salvia santolinifolia by LC-DAD-MS, Austin Chromatogr. 1, 5.

[27] M. Öztürk, M.E. Duru, B. İnce, M. Harmandar and G. Topçu (2010). A new rapid spectrophotometric method to determine the rosmarinic acid level in plant extracts, Food Chem. 123, 1352-1356.

[28] M. Coisin, R. Necula, V. Grigoraş, E. Gille, E. Rosenhech and M.M. Zamfirache (2012). Phytochemical evaluation of some Salvia species from Romanian flora, Biol. Veg. 58, 35-44.

[29] B. Tepe (2008). Antioxidant potentials and rosmarinic acid levels of the methanolic extracts of Salvia virgata (Jacq.), Salvia staminea (Montbret \& Aucher ex Bentham) and Salvia verbenaca (L.) from Turkey, Bioresour. Technol. 99, 1584-1588.

[30] G.Ş. Karatoprak, S. Ilgün and M. Koşar (2016). Antioxidant properties and phenolic composition of Salvia virgata Jacq., Turk. J. Pharm. Sci. 13, 201-212.

[31] İ.E. Poyraz, G.A. Çiftçi and N. Öztürk (2017). Phenolic contents, in vitro antioxidant and cytotoxicity activities of Salvia aethiopis L. and S. ceratophylla L. (Lamiaceae), Rec. Nat. Prod. 11, 345-355.

[32] D. Bandoniene, M. Murkovic and P.R. Venskutonis (2005). Determination of rosmarinic acid in sage and borage leaves by high-performance liquid chromatography with different detection methods, $J$. Chromatogr. Sci. 43, 372-376.

[33] I. Grzegorczyk, A. Matkowski and H. Wysokińska (2007). Antioxidant activity of extracts from in vitro cultures of Salvia officinalis L., Food Chem. 104, 536-541.

[34] M. Shekarchi, H. Hajimehdipoor, S. Saeidnia, A.R. Gohari and M.P. Hamedani (2012). Comparative study of rosmarinic acid content in some plants of Labiatae family, Pharmacogn. Mag. 8, 37-41.

[35] H. Wang, G.J. Provan and K. Helliwell (2004). Determination of rosmarinic acid and caffeic acid in aromatic herbs by HPLC, Food Chem. 87, 307-311.

[36] M. Dent, D. Bursać Kovačević, T. Bosiljkov and V. Dragović-Uzelac (2017). Polyphenolic composition and antioxidant capacity of indigenous wild Dalmatian sage (Salvia officinalis L.), Croat. Chem. Acta. 90, 451-459.

[37] G. Janicsák, I. Máthé, V. Miklóssy-Vári and G. Blunden (1999). Comparative studies of the rosmarinic and caffeic acid contents of Lamiaceae species, Biochem. Syst. Ecol. 27, 733-738.

[38] M. Modarres, J. Asili, M. Lahouti, A. Gangali, M. Iranshahy and A. Sahebkar (2014). Simultaneous determination of rosmarinic acid, salvianolic acid B and caffeic acid in Salvia leriifolia Benth. root, leaf and callus extracts using a high-performance liquid chromatography with diode-array detection technique, J. Liq. Chromatogr. Relat. Technol. 37, 1721-1730.

[39] B. Tepe, O. Eminagaoglu, H.A. Akpulat and E. Aydin (2007). Antioxidant potentials and rosmarinic acid levels of the methanolic extracts of Salvia verticillata (L.) subsp. verticillata and $S$. verticillata (L.) subsp. amasiaca (Freyn \& Bornm.) Bornm, Food Chem. 100, 985-989. 
[40] O. Yumrutas, A. Sokmen and N. Ozturk (2011). Determination of in vitro antioxidant activities and phenolic compounds of different extracts of Salvia verticillata ssp. verticillata and spp. amasiaca from Turkey's flora, J. Appl. Pharm. Sci. 1, 43-46.

[41] S. Rungsimakan and M.G. Rowan (2014). Terpenoids, flavonoids and caffeic acid derivatives from Salvia viridis L. cvar. Blue Jeans, Phytochemistry 108, 177-188.

[42] M. B. Bahadori, B. Asghari, L. Dinparast, G.n Zengin, C. Sarikurkcu, M. Abbas-Mohammadi and S. Bahadori (2017). Salvia nemorosa L.: A novel source of bioactive agents with functional connections, LWT - Food Sci. Technol. 75, 42-50.

[43] G. Zengin, E.J. Llorent-Martínez, M.L. Fernández-de Córdova, M.B. Bahadori, A. Mocan, M. Locatelli and A. Aktumsek (2018). Chemical composition and biological activities of extracts from three Salvia species: S. blepharochlaena, S. euphratica var. leiocalycina, and S. verticillata subsp. amasiaca, Ind. Crops Prod. 111, 11-21.

[44] G. Hao, H. Ji, Y. Li, R. Shi, J. Wang, L. Feng and L. Huang (2012). exogenous ABA and polyamines enhanced salvianolic acids in hairy roots cultures of Salvia miltiorhiza Bge.f.alba, Plant Omics J. 5, 446-452.

[45] T. Wang, H. Zhang, L. Wang, Y. Jiang, L. Zhang, Y. Zhou, R. Yang, Ch. Ding and X. Wang (2014). A simple and reliable method for distinguishing danshen in Salvia: simultaneous quantification of six active compositions by HPLC, J. Chromatogr. Sci. 52, 992-998.

[46] H. Dai, C. Xiao, H. Liu and H. Tang (2010). Combined NMR and LC-MS analysis reveals the metabonomic changes in Salvia miltiorrhiza Bunge induced by water depletion, J. Proteome Res. 9, $1460-1475$

[47] X.-B. Li, W. Wang, G.-J. Zhou, Y. Li, X.-M. Xie and T.-S. Zhou (2012). Production of salvianolic acid $\mathrm{B}$ in roots of Salvia miltiorrhiza (Danshen) during the post-harvest drying process, Molecules 17, 23882407.

[48] H.-S. Ho, R.K. Vishwakarma, E.C.-F. Chen and H.-S. Tsay (2013). Activation tagging in Salvia miltiorrhiza can cause increased leaf size and accumulation of tanshinone I and IIA in its roots, Bot. Stud. 54, 37.

[49] P.C. Santos-Gomes, R.M. Seabra, P.B. Andrade and M. Fernandes-Ferreira (2003). Determination of phenolic antioxidant compounds produced by calli and cell suspensions of sage (Salvia officinalis L.), J. Plant Physiol. 160, 1025-1032.

[50] O.Y. Celiktas, E. Bedir and F.V. Sukan (2007). In vitro antioxidant activities of Rosmarinus officinalis extracts treated with supercritical carbon dioxide, Food Chem. 101, 1457-1464.

[51] J. Luis and C. Johnson (2005). Seasonal variations of rosmarinic and carnosic acids in rosemary extracts. Analysis of their in vitro antiradical activity, Span. J. Agric. Res. 3, 106-112.

[52] S. Albu, E. Joyce, L. Paniwnyk, J. Lorimer and T. Mason (2004). Potential for the use of ultrasound in the extraction of antioxidants from Rosmarinus officinalis for the food and pharmaceutical industry, Ultrason. Sonochem. 11, 261-265.

[53] X. Liu, J. Du, Y. Ou, H. Xu, X. Chen, A. Zhou, L. He and Y. Cao (2013). Degradation pathway of carnosic acid in methanol solution through isolation and structural identification of its degradation products, Eur. Food. Res. Technol. 237, 617-626.

[54] F.N. Lugemwa (2012). Extraction of betulin, trimyristin, eugenol and carnosic acid using water-organic solvent mixtures, Molecules 17, 9274-9282.

[55] M. Ben Farhat, A. Landoulsi, R. Chaouch-Hamada, J. A. Sotomayor and M. J. Jordán (2013). Characterization and quantification of phenolic compounds and antioxidant properties of Salvia species growing in different habitats, Ind. Crops Prod. 49, 904-914.

[56] W. Liang, W. Chen, L. Wu, S. Li, Q. Qi, Y. Cui, L. Liang, T. Ye and L. Zhang (2017). Quality evaluation and chemical markers screening of Salvia miltiorrhiza Bge. (Danshen) based on HPLC fingerprints and HPLC-MS ${ }^{\mathrm{n}}$ coupled with chemometrics, Molecules 22, 478.

[57] X. Zhang, Y. Yu, Y. Cen, D. Yang, Z. Qi, Z. Hou, S. Han, Z. Cai and K. Liu (2018). Bivariate correlation analysis of the chemometric profiles of Chinese wild Salvia miltiorrhiza based on UPLCQqq-MS and antioxidant activities, Molecules 23, 538.

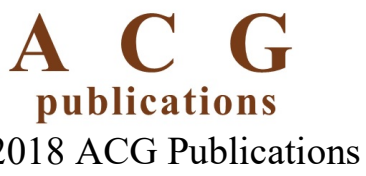

